\title{
Molecular diagnostic testing: Alphabet soup with a side of codes
}

\author{
Wendy S. Schroeder, Michael J. Demeure, Sherri Z. Millis \\ Ashion Analytics ${ }^{T M}$, Phoenix, AZ, United States
}

Received: December 1, 2015

Accepted: December 20, 2015

Online Published: January 20, 2016

DOI: $10.5430 /$ jha.v5n2p 88

URL: http://dx.doi.org/10.5430/jha.v5n2p88

\begin{abstract}
Effective and affordable health care depends on the availability of accurate, reliable and clinically valid monitoring tests. Faulty tests can lead to misdiagnosis or failure to diagnose, resulting in patients receiving unnecessary treatment, delays in treatment or no treatment when treatment is needed. Safe and effective genomic testing is increasingly more important with advances in precision/personalized medicine. The rapid evolution of technology and molecular testing has brought new hope to patients and new pressures to payors. While genomic panel testing is time and cost efficient, minimally disruptive for patients and physicians, and spares valuable specimens, payors struggle with evidence-based approaches to coverage determinations in an environment where clinical treatment options cannot keep pace with technology. The result: a highly complex coding structure, disparate coverage policies, and extremely variable reimbursement for genomic testing. Providing the right treatment to the right patient at the right time depends on meaningful tests proven to impact clinical decisions, integrated with the most current data relevant to the practice of medicine, and recognized as medically necessary to tailor treatment for the unique biology of a disease. An understanding of the test reimbursement landscape is critical to implementation of a successful personalized medicine business model.
\end{abstract}

Key Words: Molecular, Genomic, Billing, Reimbursement, Coverage, Current Procedural Terminology codes, Next generation sequencing

\section{INTRODUCTION}

1.1 Molecular diagnostic tests, personalized medicine and reimbursement

Implementation of personalized medicine and human genome sequencing into clinical practice has created a paradigm shift in the business of medicine. Technological contributions to an exponentially increasing set of genomic data outpaces scientific analysis of the data for clinical utility. The current "less is more" reimbursement model allows payment for a limited number of genes with authoritative evidence to support direct associations with response to therapy. Yet, many genes with potential therapy indications are known to interact by association in biological pathways and may be valuable in treatment planning suggesting "more is better". Clinicians and patients are aware of the value of genomic data in treatment decisions, however, reimbursement is typically limited to genes with proven clinical utility and supported by professional practice guidelines such that "more" genomic data as it is available today with large next generation sequencing (NGS) panels has been identified as a non-covered service by payors such as medical insurance companies. This analysis will first provide an overview of molecular diagnostic tests with a focus on next generation sequencing, then describe the evolution of the billing, coding, and reimbursement models, and finally, will address the current issues in managing a business model that mitigates

\footnotetext{
${ }^{*}$ Correspondence: Wendy S. Schroeder; Email: schroeder.wendy@gmail.com; Address: Ashion Analytics ${ }^{\mathrm{TM}}$, Phoenix, AZ, United States.
} 
billing risk and optimizes fair market value reimbursement from both private and government payors.

Molecular diagnostic testing requires a sample (or biopsy) from a cancerous tumor and in some cases a non tumor sample (blood or tissue) to perform a variety of in vitro diagnostic (IVD) tests. Unlike simple tests which measure a single analyte like potassium levels, molecular tests are more complex and detect or measure numerous analytes (e.g. DNA variant analysis). Molecular profiling typically involves traditional anatomical pathology and newer molecular techniques, combined with high-tech instrumentation and software. The results of molecular profiling combined with clinical interpretations identify genetic or genomic disease drivers and actionable targets (biomarkers) that are useful in diagnosis, prognosis, or influence therapy.

Molecular profiling of patients may help clinicians optimize the standard of care so patients get the most out of available therapies. To date, nineteen therapies with associated companion diagnostics relative to specific genomic alterations are Food and Drug Administration (FDA)-approved for oncology treatments. Additionally, more than 70 FDA-approved therapies have been developed that target biomarkers altered in cancer. The evidence to support the efficacy of these therapies, when targeting the specific aberrations, either on or off indication, is accumulating rapidly. Testing a patient's cancer to assess their unique tumor profile and matching their genomic alterations with the most current scientific evidence allows clinicians to identify agents with predicted clinical benefit and/or a lack of benefit, facilitating in choosing the right drug for the right patient at the right time.

NGS is a high throughput, rapid method to sequence DNA samples, used to sequence entire genomes, or to rapidly examine and more broadly detect mutations across hundreds of genomic hotspots. Evaluation of key genes may yield genomic variant information that is proven to be associated with a targeted therapy, along with variants that may be linked to treatments under development and in clinical trials, and variants of unknown significance (VUS). Many payors have determined that NGS can generate extensive genomic information, some of which has not been fully vetted for clinical utility, creating the risk of potential harm if ineffective therapy is given based on test results.

The American Medical Association (AMA) developed specific genomic sequencing procedure (GSP) codes (8141081471 ) for billing targeted genomic panels, and payors expect providers to bill using these codes when the services meet the code definition, rather than adjusting coding methodology (such as billing for individual analytes or biomarkers using tiered codes) for the purposes of payment. ${ }^{[1]}$ However, both government and private payors generally deny coverage for CPT code 81455 (large genomic sequencing panel greater than 50 genes), because while a single NGS panel may include analytes proven to provide positive patient outcomes, the panel may also include analytes for which the potential value of outcomes has not yet been proven. ${ }^{[2,3]}$ As such, payors have identified covered biomarkers for specific diagnoses and recommended billing using a tiered coding methodology that better communicates medical necessity. ${ }^{[3,4]}$ There may be considerable false claims risk associated with billing the government for targeted genomic panels that have not been deemed medically necessary, and providers are almost certain to receive denials for any claims submitted with a GSP code.

For most patients, coverage is equivalent to access to services intended to help guide treatment especially for patients with refractory cancer. If payors do not reimburse these services, providers cannot cover costs, and patients who may benefit from the new genomic testing technologies are potentially denied access based on their ability to pay. Only those relatively few patients with sufficient financial resources will have access to the most modern care. Laboratories that provide the testing services rely on nimble payor coverage policies in response to rapidly emerging technologies to be sustainable business entities. In supporting a personalized medicine business model, providers must consider billing and coding guidance to identify the most efficient coding strategy with the best FMV reimbursement opportunities and the least amount of risk.

\subsection{Laboratory developed tests and regulations}

\subsubsection{Clinical Laboratory Improvement Amendments (CLIA)}

Understanding the regulatory framework for laboratory tests is critical when considering the best strategy for NGS billing. The Centers for Medicare \& Medicaid Services (CMS), through the CLIA, regulates all laboratory testing (except research) performed on humans in the United States. CLIA oversees the labs' processes, NOT the tests that are developed. The objective of the CLIA program is to ensure quality laboratory testing. CLIA regulates requirements for establishing and maintaining quality laboratory operations and staffing with qualified personnel. CLIA regulations do not require FDA premarket review of tests or any evidence that a test accurately identifies or predicts a patient's clinical status.

Clinical laboratories must be properly certified to receive Medicare or Medicaid payments. CLIA program certification requires application and inspection. In lieu of CLIA certification, laboratories may be granted a Certificate of Accreditation from one or more of the six CMS approved 
accreditation programs. Alternatively, a laboratory may be exempt from CLIA certification when licensed in a state program established by law from CLIA program requirements (i.e. New York State).

\subsubsection{The College of American Pathologists (CAP)}

The CAP is a nonprofit organization of board-certified pathologists established to enhance the specialty practice of pathology with laboratory standards, education, and research. CAP uses a practicing professionals, peer-based inspection model to perform on-site laboratory inspections for accreditation and maintenance compliance assessments every 2 years. CMS granted the CAP Laboratory Accreditation Program (considered more stringent than CLIA) deeming authority, which allows CAP inspection in lieu of a CMS (CLIA) inspection. CAP accreditation is also recognized by The Joint Commission and can be used to meet many state certification requirements.

\subsubsection{Interagency (CMS and FDA) framework}

The FDA oversees safety and efficacy of lab developed tests (LDTs) and IVDs and recognizes the evolution of LDTs from relatively low risk, simple tests to advanced and complex technologies now used by clinicians to make treatment decisions. The FDA is obligated to assure these tests are accurate, reliable and clinically meaningful. Although the roles of the agencies are different, FDA and CMS share an interest in LDT oversight to ensure public confidence in accurate and clinically meaningful tests while avoiding unnecessary regulatory burden or redundancy.

\section{Medical device regulations}

The Social Security Act qualifies a billable service as one which: (1) falls within a Medicare benefit category, (2) is not otherwise excluded from coverage, and (3) is reasonable and medically necessary. ${ }^{[5]}$ In 1976 , Congress enacted the Medical Device Amendments (MDA) to regulate medical devices used in humans, including IVDs. The application of these regulations relative to FDA requirements for safety and efficacy was clarified in the Category B device regulations in 1995.

While medical devices work on or in a person, IVDs are used to collect, prepare or examine specimens after they are removed from the body. They include reagents, instruments, and systems intended to diagnose disease or determine a state of health. IVDs are exempt from the Category B Investigational Device Exemption (IDE) regulations if the IVD: (1) has been in commercial distribution since prior to the 1976 device amendments, or (2) it is substantially equivalent to a pre-amendment device and is used according to its labeled indications, or (3) when considered for research is not used as a diagnostic procedure without confirmation by another medically established criteria, is non-invasive, and is properly labeled. ${ }^{[6]}$

If the IVD does not fit into one of these 3 categories, and it is considered significant risk because inaccurate IVD results could lead to misdiagnosis and/or treatment error, the IVD is subject to the medical device regulations and an approved IDE is required to conduct research in support of an FDA review for safety and effectiveness. ${ }^{[6]}$ An example of FDA enforcement of device regulations in the IVD space is noted in a recent FDA warning letter indicating CancerIntercept ${ }^{\mathrm{TM}}$ meets the definition of a medical device in that it is a non-invasive blood test used to screen for early detection of cancer. ${ }^{[7]}$ Their letter informs the manufacturer that FDA believes the vendor is offering a high risk test that has not been clinically validated and as such the test may be harmful to the public.

\section{FDA Draft guidance on oversight of LDTs}

Since 1988, CMS has regulated laboratories, including those that develop LDTs, under CLIA (42 U.S.C. 263a). CLIA governs the accreditation, inspection and certification process for laboratories. CLIA requirements address the accuracy and reliability of laboratory testing processes. Under CLIA, accreditors do not evaluate the accuracy with which specific LDTs identify, measure, or predict the presence or absence of a clinical condition or predisposition in a patient (clinical validation). "The FDA assures both the analytical validity (e.g., analytical specificity and sensitivity, accuracy and precision) and clinical validity of diagnostic tests through its premarket clearance or approval process... In addition to premarket review, FDA requirements provide other controls to ensure appropriate design, manufacture, and safety and effectiveness of medical devices." ${ }^{[6]}$ Until recently, the FDA has generally not enforced premarket review and other applicable FDA requirements because LDTs were relatively simple lab tests and generally available on a limited basis. Currently, however FDA has indicated that with the evolution in complexity and risk implications, CLIA oversight alone does not ensure that LDTs are properly designed, consistently manufactured, and are safe and effective for patients. FDA has issued proposed draft guidance on oversight of LDTs.

The FDA Draft guidance points out:

- The volume and complexity of LDTs has evolved considerably since 1976.

- LDTs previously involving manual techniques using legally marketed components (reagents, Immunohistochemical [IHC] stains, etc.) have transitioned to high-tech instrumentation and software to generate 
results and clinical interpretations.

- Whereas LDTs were historically used for rare diseases (potentially qualified as Humanitarian Use Devices or HUDs in the new oversight proposal) or developed to meet the needs of a local population and interpreted directly by providers responsible for the patient's care, technologically advanced devices (LDTs) are now guiding critical clinical decisions for highrisk diseases and conditions ("personalized medicine"), providing results to a wide geographic distribution and being marketed by large corporate manufacturers.

Under FDA's proposed risk-based framework for the oversight of LDTs, CMS through CLIA will continue to monitor laboratory operations including the testing process, and FDA will enforce compliance with the agency's quality systems regulation pertaining to the design and manufacture of laboratory tests. FDA would consider several factors when classifying LDTs by risk including:

- intended use for high risk diseases, conditions or populations

- screening or diagnostic intended use

- clinical decisions potentially made based on the test result

- clinical/treatment decisions made based on test result in combination with other information

- alternative diagnostic and treatment options available to the patient

- potential consequences/impact of inaccurate and/or erroneous results

- the number and type of adverse events associated with the device ${ }^{[6]}$

Because of the extremely large universe of variants, especially in whole exome or whole genome sequencing, predefining a specific NGS device intended use in a PMA submission for an IVD determined to be high risk is unrealistic. As such, FDA is investigating a performance standardsbased approach to regulating NGS in the context of their proposed LDT risk-based oversight framework. Key opinion leaders suggest instrumentation, reagents, and regulation of databases and variant interpretation will be addressed in the final guidance. ${ }^{[8]}$

\section{Methods}

\subsection{Billing, coding and reimbursement assessment}

New AMA GSP codes for genomic sequencing panels seemed to establish codes for NGS genomic panels that would serve to communicate the provision of this healthcare service between providers and payors. Unfortunately,

Published by Sciedu Press
CMS did not assign pricing for these codes in the clinical laboratory fee schedule (CLFS), and payors began to establish policies that explicitly denied payment for GSP codes. We looked at molecular diagnostic and NGS reimbursement experiences, retained a healthcare consulting firm with expertise in coding and reimbursement, and performed a comprehensive review of government coverage decisions and private payor policies for NGS, to equip us with information needed to identify the most efficient coding strategy with the best FMV reimbursement opportunities and the least amount of risk.

\subsection{Molecular diagnostics technology evaluation}

Multiple IVD technologies may be used to perform molecular testing and are summarized in the following bullets:

- IHC: a semiquantitative method of analyzing and identifying cell types based on the binding of labelled antibodies to specific cell components to determine level of protein expression.

- Chromogenic/Fluorescence in situ Hybridization (CISH/FISH): cytogenetic technique used to detect the localization or copy number of specific DNA sequences or genes on chromosomes; detects gene deletions, amplifications, translocations and fusions using fluorescent or chromogenic dyes; requires the use of a specialized microscope to visualize the cells.

- Microarray: Hybridization technique that allows simultaneous detection of a large number of targets (SNPs [Single-nucleotide Polymorphism], mRNA or DNA sequences) from the same sample in a short span of time.

- Polymerase Chain Reaction (PCR) and Reverse Transcription (RT)-PCR: Nucleic acid amplification technique that selective isolates and amplifies specific DNA fragments.

- Quantitative Polymerase Chain Reaction (qPCR): amplifies and quantifies a targeted DNA molecule.

- Sanger Sequencing: examines strands of DNA to identify mutations by analyzing long contiguous sequencing reads.

- NGS: a high throughput method to sequence DNA and RNA samples, used to determine the sequence of entire genomes, exomes or hundreds of hotspots within genomes.

- Various methods to measure post-translational modifications, such as Phosphorylation, a post translation modification of a protein which causes it to become a target for binding or interacting with other proteins, or Methylation. 
An IVD may be FDA approved as its own unique medical device. For example, in May of 2015 the FDA approved the Cobas $₫$ RT-PCR KRAS Mutation Test for diagnostic use. The real-time PCR test (performed on the Cobas 4800 System, including amplification, detection and software automation for interpretation and reporting) is designed to identify KRAS mutations in tumor samples from metastatic colorectal cancer (mCRC) patients and aid clinicians in determining a therapeutic path for them. Cobas 4800 System approvals in the U.S. also include the the Cobas BRAF V600 Mutation Test and the Cobas EGFR Mutation Test.

Alternatively, an IVD may be FDA approved contemporaneously with a related therapeutic product. An IVD or imaging tool that provides information essential for the safe and effective use of a corresponding therapeutic product is called a companion diagnostic device. Clinical trials for these products are designed to demonstrate the IVD produces reliable and repeatable test results and have an association with utility in the safe and effective use of the drug. The approved use of both the IVD and the drug is stipulated in the product labeling and instructions for use for both the device and the drug. Two examples are described herein.

The Cobas 4800 BRAF V600 Mutation Test is an IVD device intended for the qualitative detection of the BRAF V600E mutation in DNA extracted from formalin-fixed, paraffinembedded human melanoma tissue. The Cobas 4800 BRAF V600 Mutation Test is a real-time PCR test on the Cobas 4800 system, and is intended to be used as an aid in selecting melanoma patients whose tumors carry the BRAF V600E mutation for treatment with vemurafenib. ${ }^{[9]}$

Zelboraf $^{\mathrm{TM}}$ (Vemurafinib) is a kinase inhibitor indicated for the treatment of patients with unresectable or metastatic melanoma with BRAF V600E mutation as detected by an FDA-approved test. Limitation of Use: ZELBORAF is not recommended for use in patients with wild-type BRAF melanoma. ${ }^{[10]}$

A current list of companion diagnostics that are FDA approved is available at http://www.fda.gov/MedicalDev ices/ProductsandMedicalProcedures/InVitroDia gnostics/ucm301431.htm.

Another molecular diagnostic testing technology, multianalyte assays with algorithmic analyses (MAAA), are considered advanced diagnostic laboratory tests offered and furnished by a single laboratory and not sold for use by a laboratory other than the original developing laboratory. These tests, if not cleared or approved by the FDA, must be limited to an analysis of multiple biomarkers of DNA, RNA or proteins combined with a unique algorithm to yield a single patient-specific classification, score or index (e.g. Oncotype DX $\AA$ Colon Cancer Assay, Genomic Health [CPT 81525], ChemoFX $囚$, Helomics, Corp. [CPT 81535 and 81536 for each additional single drug or drug combination], CancerTYPE ID, bioTheranostics, Inc. [CPT 81540], Afirma $(R$ Gene Expression Classifier, Veracyte, Inc. [CPT 81545], and others).

Lastly and most recently introduced into the marker are NGS panels, which often generate gene variant reports, with interpretation and targeted therapy associations. While much of the analysis is done by algorithms and/or software, there is still a component of clinical expert judgment in generating the report that goes out to the physician.

\subsection{Billing and reimbursement historical context}

Medicare covers statutory categories of healthcare including diagnostic tests. Prognostic tests are not covered. Diagnostic tests under Medicare must contribute to the diagnosis or management of disease such that screening tests and tests performed in the absence of personal signs or symptoms, are generally not covered. Likewise, a familial history with a $25 \%-50 \%$ chance of a more serious problem is not considered a personal sign or symptom supporting medical necessity. Pathology and clinical laboratory tests are "bundled" with Medicare's inpatient DRG (diagnosis related group) payment such that molecular testing on blood or tissue samples of inpatient origin must be paid by the hospital from its DRG reimbursement while the patient is an inpatient and up to 14 days after discharge or 30 days after biopsy whichever comes first ("14-day rule").

The Social Security Act is the overarching statute that mandates no Medicare (CMS) payment may be made for any expenses incurred for items and services which are not reasonable and medically necessary. CMS has historically required evidence of safety and efficacy (e.g. FDA labeling) as a requirement to determine medical necessity. FDA-regulated products must receive FDA approval or clearance (unless exempt from the FDA premarket approval review process) for at least one indication to be eligible for Medicare coverage; however, FDA approval/clearance alone does not generally equate to Medicare coverage. CMS bases coverage decisions on authoritative publications or alternatively generally accepted medical practice as evidence the items and services are reasonable and medically necessary to treat illness or injury, not experimental.

"On March 10, 2000, a proposed rule was published in the Federal Register (65 FR 13082) that set forth uniform national coverage and administrative policies for clinical diagnostic laboratory services. These proposed policies reflected 
the consensus of the Negotiated Rulemaking Committee. The final rule, published in the Federal Register on November 23, 2001 (66 FR 58788), addresses the public comments received on the proposed rule. The final rule established the national coverage and administrative policies for clinical diagnostic laboratory services payable under Medicare Part B. It promotes Medicare program integrity and national uniformity, and simplifies administrative requirements for clinical diagnostic services. There are 23 national coverage determinations included in the final rule...". ${ }^{[1]}$

CMS issues National Coverage Decisions (NCDs) about coverage for specific medical items, services, treatment procedures, or technologies. In the absence of a specific NCD, the Medicare administrative contractor (MAC) is responsible for determining whether an item or service is reasonable and necessary. A local coverage determination (LCD) is a decision by a MAC on whether to pay for a particular service. MACs provide coverage decisions on a contractorwide basis by issuing an LCD. Contractors may restrict a "medically necessary" coverage decision to specific diseases or diagnoses (ICD-10-CM codes) and may require supporting documentation such as personal, medical, and family history information consistent with the local policy. The billing provider may be required to capture the diagnosis or condition on a requisition.

CMS considers professional practice guidelines (e.g. National Comprehensive Cancer Network [NCCN], American Society of Clinical Oncology [ASCO], etc.) as authoritative evidence useful to support medical necessity when no coverage determinations exist. Individual testimonials and limited case studies are not considered to be a broad enough evidence to support generally accepted medical practice or medical necessity. Billing for reasonable and medically necessary items and services not specifically addressed by an NCD or an LCD should be based on:

(1) Evidence from randomized or other robust clinical trials released in authoritative publications;

(2) Generally accepted professional practice guidelines supported by rigorous medical evidence based on:

- peer-reviewed medical journal publications of scientific data or research studies;

- consensus of expert medical opinion (i.e., recognized authorities in the field); or

- medical opinion derived from consultations with medical associations or other health care experts.

Billing for services that are not reasonable and medically necessary is a false claim. The Civil False Claims Act (FCA) imposes liability on a person or entity who knowingly (reck- less disregard or deliberate ignorance) submits a false claim. FCA violations carry penalties of not less than $\$ 5,000$ and up to $\$ 10,000$ per each false claim and 3 times the damages to the federal health program. Criminal false claims imposes potential liability on an individual such as the Chief Executive Officer (CEO), Chief Financial Officer (CFO), or Chief Operations Officer (COO) who makes a false claim, or conceals or fails to disclose false claims events with an intent to commit fraud. Criminal penalties include fines of up to $\$ 25,000$, and 5 years in prison. In both situations, individuals and entities may also be excluded from participation in government funded programs. At least 23 states have false claims acts that may also restrict government claims submissions for molecular testing.

Medicare coverage rules are based on prior authority, laws and statutes with potential application to any government payor (Medicaid, PHS grants, federal flow-through dollars to States or Universities), and many private payors adopt Medicare coverage decisions. Private insurers are looking for effective technology and tests that improve outcomes for patients (clinical utility) and may manage benefits and co-pays based on benefit categories such as screening, preventive and diagnostic services (may be regulated by state-to-state requirements, e.g. all states require private insured plans to cover mammography while coverage for PSA and colorectal cancer screening varies from state to state). There are no state mandates related to complex diagnostics for personalized medicine.

\subsection{CPT coding historical context}

"The HIPAA act required the Secretary of Health and Human Services (HHS) to establish standard code sets for transmitting healthcare services data between providers and payors." ${ }^{[12]}$ Regulations were finalized in 2000 and established the AMA Current Procedural Technology (CPT) system with CPT codes for physician services and laboratory tests, and ICD-9-CM (transitioned to ICD-10-CM as of October 1, 2015) codes for diseases. The AMA's CPT editorial panel assigns CPT codes based on evidence required to determine the appropriateness of the code.

\subsubsection{Tiered Molecular Pathology (MoPath) codes}

Historically, molecular diagnostic tests were reimbursed through a method known as "code stacking" according to process steps (not by analyte) where each step or process used to perform a test was billed and reimbursed. Between 2010 and 2012, a tiered coding system was developed to link genes with the intended use of a test. Tier 1 MoPath CPT codes (81200-81383) are used for the majority of commonly performed single-analyte molecular tests (Example: testing for BRAF V600E variants is billed using CPT Code 81210). 
Tier 2 MoPath codes (81400-81408) represent tests generally performed in lower volumes than Tier 1 procedures and are arranged by nine levels of technical resources and interpretive work performed by the physician or other qualified health care professional (Example: CPT Code 81404 is used to bill 2-5 exons; 81405 for 6-10 exons; 81406 for 11-25; 81407 for 26-50; and 81408 for $>50$ exons). MoPath codes include all aspects of sample preparation, cell lysis, internal measures to assure adequate quantity of DNA or RNA, and performance of the assay.

In 2013, CMS announced it would restructure the clinical laboratory reimbursement process and assigned individual CPT codes to more than 100 existing tests. CPT code 81479 Unlisted or Generic test code was introduced in 2013 for molecular pathology procedures not specified by Tier 1 and Tier 2 codes. In 2014, no approved single molecular pathology codes for large gene/genomic panels/NGS assays existed. Coding guidelines did not clearly explain how to report expanded panel testing when all analytes billed were performed on a single patient specimen.

\subsubsection{GSP codes}

The AMA established a framework for GSPs and a coding structure CPT 81410-81471, effective, January 1, 2015. Codes designated for NGS testing include:

- CPT 81445 - Targeted genomic sequence analysis panel, solid organ neoplasm, DNA analysis, 5-50 genes interrogation for sequence variants and copy number variants or rearrangements, if performed.

- CPT 81450 - Targeted genomic sequence analysis panel, hematolymphoid neoplasms or disorders, DNA analysis, 5-50 genes interrogation for sequence variants and copy number variants or rearrangements, if performed.

- CPT 81455 - Targeted genomic sequence analysis panel, solid organ or hematolymphoid neoplasm, DNA and RNA analysis when performed; 51 or greater genes interrogation for sequence variants and copy number variants or rearrangements, if performed.

\subsection{Current status of coverage for molecular diagnostic tests}

A national coverage policy document for diagnostic laboratory tests states CMS's policy with respect to the clinical circumstances in which the services will be considered reasonable and necessary, and not screening for Medicare purposes.

"Services excluded from coverage include routine physical examinations and other services that are not reasonable and necessary for the diagnosis or treatment of an illness or injury. CMS interprets these provisions to prohibit coverage of "screening" services, including laboratory test services furnished in the absence of signs, symptoms or personal history of disease or injury, except as explicitly authorized by statute. A test service might be considered medically appropriate, but nonetheless excluded from medical coverage by statute [NCDs] apply nationwide. A national coverage policy is neither a practice parameter nor a statement of the accepted standard of medical practice."[11]

Likewise, private payors manage benefits based on categories such as screening, preventive and diagnostic services and tend to adopt Medicare policy.

\subsubsection{Marker or technology specific coverage}

A number of government LCDs address marker or technology specific coverage (summarized in Table 1). Most private payors also have policies with similar coverage statements. For example, CIGNA has unique policies for testing circulating tumor cells, gene-based prostate cancer screening, detection, and disease monitoring, breast cancer prognosis genetic expression assays; genetic susceptibility to breast and ovarian cancer (e.g. BRCA1 and BrCA2); pharmacogenetic testing; PSA screening for prostate cancer; and tumor in vitro chemosensitivity and chemoresistance assays.

\subsubsection{Government payor coverage for large panel (NGS) tests}

During ongoing CMS deliberations aimed at determining coverage and a permanent pricing structure for molecular diagnostic tests in 2014, interim pricing using a method known as gapfill triggered a requirement for interactive negotiation between providers (billing sites) and MACs. As such there is no general comment NCD for molecular diagnostic test, gene/genomic panels, or NGS tests. CMS annually publishes a file of test codes and interim prices for clinical diagnostic laboratory tests determined to be priced by MACs using the gapfilling methodology. Reasons for blank pricing for a given code by a specific MAC may include:

- There is no benefit category for the test.

- There is no high quality evidence published in the peer reviewed literature demonstrating clinical utility for the Medicare population.

- There is a lack of medical necessity: knowing the exact genetic sequencing will not change the treatment or prognosis of a beneficiary.

- The MAC has not received a technical assessment that qualifies the test for coverage.

While no NCD specific for molecular diagnostic or NGS tests exists, all but one MAC has issued LCDs on molecular diagnostic testing with reference to NGS panels. There are more 
than 2800 MAC LCDs, approximately 20 of which have a direct relevance to targeted gene/genomic panels/NGS. Rather than relying on a keyword search of the CMS database to yield a comprehensive listing of LCDs with content addressing molecular testing, we looked at every LCD using the alphabetized index to identify these 20 LCDs with implications for gene/genomic panels. Relevant content from these MAC LCDs (including coding guidance) is summarized (see Table 2).

Table 1. Marker/technology specific LCDs as accessed from CMS coverage database Nov 2015 ${ }^{[4]}$

\begin{tabular}{|c|c|}
\hline Marl & overage Statement \\
\hline $\begin{array}{l}\text { Circulating Tumor } \\
\text { Cells Assays }\end{array}$ & $\begin{array}{l}\text { Including but not limited to CellSearch (Veridex), OncoCEE (Biocept) and PCR (RT-PCR) assays are not proven effective for the diagnosis or } \\
\text { treatment of breast, colorectal, or prostate cancer. CellSearch }{ }^{\circledR} \text { Circulating Tumor Cell (CTC) (Veridex, LLC) assay is covered (frequencies } \\
\text { specified) for metastatic breast, colorectal, and prostate. All other methods for CTC detection, including PCR (RTPCR) assays, are } \\
\text { non-covered. Four contractors allow an exception with limited coverage for the CellSearch }{ }^{\circledR} \text { Circulating Tumor Cell (CTC) (Veridex, LLC) } \\
\text { assay for metastatic breast, colorectal, and prostate. (All other methods for CTC detection, including PCR (RTPCR) assays, are non-covered.) }\end{array}$ \\
\hline $\begin{array}{l}\text { Breast/Ovarian Cancer } \\
\text { Testing }\end{array}$ & $\begin{array}{l}\text { Including Breast Cancer Gene Expression Ratio, MammaPrint }{ }^{\circledR} \text {, Rotterdam 76-Gene Signature, the } 41 \text {-gene signature assay, Amsterdam } \\
\text { 70-Gene Profile, OVA-1 and the ROMA TM test are not covered in the diagnosis or treatment of breast/ovarian cancer. There are some contractor } \\
\text { exceptions with limited coverage based on specified criteria for: } \\
\text { - BRCA } 1 \text { and BRCA } 2 \text { genetic testing }(81211,81212,81213,81215,81217) \\
\text { - the Breast Cancer Index (aka BCI, bioTheranostics }(81479) \text { ) } \\
\text { - Prosigna breast cancer gene signature (CPT Code } 0008 \mathrm{M} \text { oncology (breast), MRNA Analysis of } 58 \text { genes using hybrid capture, on } \\
\text { FFPE tissue, prognostic algorithm reported as a risk score) } \\
\text { - Oncotype DX }{ }^{\mathrm{TM}} \text { (CPT Code } 81519 \text { : Oncology (Breast), MRNA, Gene expression profiling by RT-PCR of } 21 \text { genes using FFPE tissue, } \\
\text { algorithm reported as a score) } \\
\text { Coverage of CA-125 is allowed by the national coverage decision NCD 190.28. }\end{array}$ \\
\hline Genetic Testing & $\begin{array}{l}\text { Is covered (with limitations) in a stepped approach for Lynch Syndrome (CPT code } 81210 \text { BRAF, } 81292-81300 \text { Mlh1 gene full seq-Msh6 gene } \\
\text { dup/delete variant, } 81317-81319 \text { Pms } 2 \text { gene full seq analysis - Pms } 2 \text { gene dup/delet variants, } 81403 \text { Mopath procedure level } 4 \text { and other IHC } \\
\text { codes), however universal testing for colorectal and endometrial cancers by MSI/MMR protein expression by IHC is not a Medicare benefit. } \\
\text { NRAS testing ( } 81404 \text { - molecular pathology procedure, Level } 5 \text { (e.g. analysis of } 2-5 \text { exons by DNA sequence analysis, mutation scanning or } \\
\text { duplication/deletion variants of 6-10 exons, or characterization of dynamic mutation disorder/triplet repeat by southern blot analysis)) is covered } \\
\text { for metastatic colorectal cancer, per NCCN guidelines (Version 3.2014). NRAS testing for melanoma is not covered. } \\
\text { Genetic testing of the CYP2C19 gene is covered only for patients with Acute Coronary Syndrome (ACS) undergoing Percutaneous Coronary } \\
\text { Intervention (PCI) on Clopidogrel (Plavix) therapy. CYP2D6 gene is covered to guide medical treatment and/or dosing for individuals on } \\
\text { amitryptyline or nortriptyline for depression, tetrabenazine dosing greater than } 50 \text { mg/day or re-initiation of therapy with higher doses (not } \\
\text { covered for Tamoxifen). HLA-B } 15: 02 \text { genotype testing is covered with limitations for individuals of Asian descent with planned initial } \\
\text { treatment with carbamazepine, phenytoin, or fosphenytoin. }\end{array}$ \\
\hline $\begin{array}{l}\text { Epigenetic/Genomic } \\
\text { Assays }\end{array}$ & $\begin{array}{l}\text { ConfirmMDx epigenetic assay (MDxHealth, Irvine, CA) (81479) for prostate cancer is covered for patients who meet specified prostate cancer } \\
\text { criteria to reduce unnecessary repeat prostate biopsies. } \\
\text { Decipher }{ }^{\circledR} \text { prostate cancer classifier assay (GenomeDX Biosciences Corp) ( } 81479 \text { ) is covered when certain criteria are met and use is to } \\
\text { determine which patients traditionally considered high risk of recurrence after radical prostectomy (RP) may be closely followed rather than } \\
\text { receive post-operative radiation therapy (XRT). } \\
\left.\text { Oncotype DX }{ }^{\circledR} \text { Prostate Cancer Assay (Genomic Health }{ }^{\mathrm{TM}} \text { ) and Prolaris }{ }^{\mathrm{TM}} \text { prostate cancer assay (Myriad, Salt Lake City, UT) ( } 81479\right) \text { provide } \\
\text { a biologic measure of cancer aggressiveness and are covered when certain criteria are met to help determine which patients with early stage, } \\
\text { needle biopsy proven prostate cancer, can be conservatively managed rather than treated with definitive surgery or radiation therapy. }\end{array}$ \\
\hline K-ras testing & Is covered prior to epidermal growth factor receptor antibody use in colorectal cancer \\
\hline BCR-ABL testing & $\begin{array}{l}\text { For (negative) Myeloproliferative Disease is covered in a stepped approach. Reflex testing to the next gene will be considered reasonable and } \\
\text { necessary if a sequence of genetic tests produce a negative result: } 1 \text {. BCR-ABL = negative test results, progress to } 2 \text {. JAK } 2 \mathrm{cv}=\text { negative test } \\
\text { results, progress to } 3 \text { JAK, exon } 12=\text { negative test results, progress to } 4 . \mathrm{MPL} / \mathrm{CALR}\end{array}$ \\
\hline $\begin{array}{l}\text { MGMT methylation } \\
\text { analysis testing }\end{array}$ & $\begin{array}{l}\text { Is covered for high-grade malignant glioma (e.g. glioblastoma multiforme (GBM), anaplastic astrocytoma) when patients are able to tolerate } \\
\text { temozolomide therapy or radiation therapy and the physician will use the MGMT testing results to decide between radiation therapy and } \\
\text { chemotherapy alone as } 1^{\text {st }} \text { line adjuvant treatment, or between temozolomide and other chemotherapy for } 1^{\text {st }} \text { line adjuvant treatment. }\end{array}$ \\
\hline $\begin{array}{l}\text { Human Leukocyte } \\
\text { Antigen (HLA) } \\
\text { Typing (81370-81383) }\end{array}$ & $\begin{array}{l}\text { Will be considered reasonable and medically necessary for } 1 \text {. solid organ and hematopoietic stem cell/bone marrow transplant under specified } \\
\text { conditions, } 2 \text {. disease association: HLA-B } 27 \text { for the diagnosis of certain cases of symptomatic patients with presumed ankylosing spondylitis or } \\
\text { related inflammatory disease; unclear diagnosis of celiac disease and gluten hypersensitivity usually related to ambiguous standard laboratory } \\
\left.\text { results and/or inconsistent biopsy results (e.g., HLA-DQ2 by HLA-DQB } 1^{*} 02 \text { and of DQ8 by HLA-DQB } 1^{*} 0302\right) ; 3 \text {. pharmacogenetics: HLA - } \\
\mathrm{B}^{*} 5701 \text { - abacavir for HIV, HLA-B } 1502 \text { - carbamazepine therapy in the treatment of patients at high risk of having this allele. HLA-B } 1502 \\
\text { occurs almost exclusively in patients with ancestry across broad areas of Asia, including South Asian Indians; } 4 \text {. HLA compatible platelets for } \\
\text { transfusion. }\end{array}$ \\
\hline
\end{tabular}


Table 1. (continued.)

\begin{tabular}{|c|c|}
\hline Marker/Technology & Coverage Statement \\
\hline \multicolumn{2}{|l|}{ In Vitro } \\
\hline Chemosensitivity \& & Including DiSC assay, ATP (Adenosine Triphosphate) assay , MTT (Methyl Thiazolyl Tetrazolium) assay, HDRA® (AntiCancer Inc) Assay, \\
\hline Chemoresistance & EVA-PCD ${ }^{\mathrm{TM}}$ (Rational Therapeutics) assay, Oncotech EDR $®$ (Exiqon Diagnostics) are experimental and investigational and not covered. \\
\hline \multicolumn{2}{|l|}{ Assays } \\
\hline $\begin{array}{l}\text { Urinary Tumor } \\
\text { Markers for Bladder } \\
\text { Cancer }\end{array}$ & $\begin{array}{l}\text { Including Bladder Tumor Antigen Stat (BSTAstat) (86294), and Nuclear Matrix Protein } 22 \text { (NMP-22) Immunoassay for tumor antigen, } \\
\text { qualitative or semiquantitative ( } 88120 \text { cytopathology manual, } 88121 \text { cytopathology automated) is covered for initial diagnosis of hematuria } \\
\text { suspicious of bladder cancer, monitoring tumor recurrence in conjunction with cystoscopy, monitoring recurrence after one or more } \\
\text { recurrences have been treated. Urinary FISH test is not covered to provide additional confirmatory information after a diagnosis of bladder } \\
\text { cancer recurrence has already been determined and not yet treated. Urovysion to identify recurrent bladder cancer is billed using CPT codes } \\
88120 / 88121 \text {. }\end{array}$ \\
\hline Cytogenic Studies & $\begin{array}{l}\text { Molecular cytogenetic studies utilizing the method of fluorescence-in-situ-hybridization (FISH) (88365, 88367/computer assisted technology) } \\
\text { or 88368/manual) is covered for genetic disorders (e.g., mongolism) in a fetus; failure of sexual development; HER-2/neu tests on histological } \\
\text { sections of breast cancers as potentially useful prognostic information and therapeutic indications for treating metastatic disease with anti- } \\
\text { HER-2/neu antibodies; or for the differential diagnosis of lymphoid and myeloid leukemias and myelodysplastic syndromes and to determine } \\
\text { appropriate targeted therapy. }\end{array}$ \\
\hline $\begin{array}{l}\text { Flow Cytometry } \\
\text { (88182) }\end{array}$ & $\begin{array}{l}\text { Is covered for selected patients (without metastatic disease) with the following conditions: endometrial, renal cell and prostate } \\
\text { adenocarcinoma, urinary bladder and ovarian carcinoma, mediastinal neuroblastoma, medulloblastoma. Covered when prognostic information } \\
\text { will affect treatment decisions in patients with localized disease (mediastinum, uterus, ovary, prostate, bladder, kidney/renal, brain, gastric, } \\
\text { breast, colon, rectal). Usually limited to } 24 \text { markers in a panel and performed one time after a diagnosis has been made and before treatment is } \\
\text { initiated. }\end{array}$ \\
\hline $\begin{array}{l}\text { Immunohistochemistr } \\
\text { y (IHC) (88341, } \\
88342)\end{array}$ & $\begin{array}{l}\text { May be covered for the evaluation of various malignancies, for diagnosis, staging, and the estimation of prognosis. ER and PR performed by } \\
\text { IHC specifically for tamoxifen therapy, Her2 testing in esophago-gastric and gastric cancers to determine response to trastuzumab, and ER, } \\
\text { PR and Her2 testing for the purpose of identifying patients likely to respond to hormonal therapy, biologics or chemotherapy is covered when } \\
\text { medically necessary for breast and gastric adenocarcinoma }\end{array}$ \\
\hline
\end{tabular}

\subsubsection{Private payor coverage for large panel (NGS) tests}

Several private payor medical policies deem the use of certain molecular diagnostic tests, primarily expanded mutation panels for selecting targeted cancer treatment, as experimental, investigational or unproven. A limited review of private payor policies is summarized (see Table 3 ).

\section{RESULTS}

Because of the rapid evolution of technology, pending changes to regulatory oversight, and complex billing and coding guidance, consultants are not likely to give specific coding advice. The AMA developed specific GSP codes (81410-81471) for billing targeted genomic panels and, payors expect providers to bill using these codes when the services meet the code definition, rather than adjusting coding methodology (such as billing for individual analytes or biomarkers using tiered codes) for the purposes of payment. That being said, both government and private payors generally deny coverage for CPT code 81455 because, while a single NGS panel may include analytes proven to provide positive patient outcomes, the panel may also include analytes for which the potential value of outcomes has not yet been proven. As such, Panacea Healthcare Solutions reports "in their experience", providers more often bill using tiered codes to avoid using CPT code 81455 which is associated with claims denials (personal communication).

There are government and private payor precedents in LCDs/policies that identify individual biomarkers (unique Tier 1 and Tier 2 CPT codes) deemed medically necessary (covered) for specified diagnoses (ICD-9/10 codes). For example, the CMS MAC Novitas, considers payment for BRAF V600E (81210), JAK2 (81270), KRAS (81275), MLH1 (81292/81294), KRAS codon 61 (81403), NRAS (81404), PIK3CA and KRAS Codon 146 (81479), and potentially Msh6 germline mutations (81298/81299/81300) for patients with colorectal cancer (ICD-9 codes 152.0-152.9, 153.0-153.9). Likewise, Cigna's policy includes a table of covered indications and tumor markers/CPT Codes.

\subsection{Government payors}

There may be considerable false claims risk associated with billing the government for targeted genomic panels. On July 26,2011 , a diagnostic lab provider filed a request to challenge a qualified independent contractor determination that molecular profiling serves were not eligible for Medicare payment because the services were not yet proven to be clin- 
ically effective and still considered to be investigational. In December of 2011, a HHS administrative law judge (ALJ) in this case determined a certain molecular profiling panel of tests to be "effective in that it allows doctors to make educated decisions on how best to treat their patients on a long-term basis and potentially minimizes their exposure to unnecessary drugs... substantiated by... medical articles regarding the use of molecular profiling..." ${ }^{[17]}$ In February of 2012 CMS filed a motion to review this judgement with the argument that the judge erred in finding the molecular profiling services to be reasonable and medically necessary. The case was subsequently sent back to the court citing the analysis of the medical articles regarding molecular profil- ing combined with expert testimony and medical records of beneficiaries as insufficient evidence to establish the testing was reasonable and medically necessary and not experimental. The remand order references an LCD for genetic testing, L24308 and requires the ALJ to determine whether the billed services and the claim coding are allowable (reasonable and medically necessary) pursuant to the coverage decision published in this LCD (see \#3 below).

Providers must carefully weigh false claims risks associated with billing targeted gene/genomic panel tests to government payors in comparison to the potential business benefit of reimbursement considering the following:

Table 2. MAC LCDs relevant to expanded panel tests; LCDs accessed from Ref. ${ }^{[4]}$

\begin{tabular}{|c|c|}
\hline MAC (Jurisdiction) & Coverage Statement \\
\hline $\begin{array}{l}\text { Novitas (AR, CO, NM, OK, TX, } \\
\text { LA, MS, DE, DC, MD, NJ, PA) }\end{array}$ & $\begin{array}{l}\text { Specifies covered biomarkers (and CPT codes) that have proven clinical validity/utility. "Non-conventional methods of next } \\
\text { generation sequencing (NGS), which can generate much more extensive genomic information than conventional techniques, are } \\
\text { currently non-covered. NGS methods which provide more 'intermediate' range information (e.g., in the 5-50 mutation range) may be } \\
\text { performed in the laboratory, pending adequate quality control, such as CLIA certification, but the actual coding and billing will } \\
\text { continue to follow the 'one-at-a-time' biomarker approach based on this LCD." Targeted genomic sequencing (CPT codes } 81445 \text {, } \\
81450 \text {, and } 81455 \text { ) are NOT covered "because an alternative coding structure has already been established in order to best specify } \\
\text { medical necessity." }\end{array}$ \\
\hline $\begin{array}{l}\text { Wisconsin Physicians Service } \\
\text { Insurance Corporation (WPS) } \\
\text { (IA, KS, MO, NE, IN, MI) }\end{array}$ & $\begin{array}{l}\text { Covers reasonable and medically necessary genetic testing performed in a CLIA/CAP approved laboratory with appropriate state } \\
\text { licensing and a laboratory director holding a relevant doctoral degree from an accredited institution. Their policy is also limited } \\
\text { explicitly to unique tests with authoritative evidence supporting medical necessity for specific diagnosis codes. The policy does not } \\
\text { address GSP codes }(81450,81445 \text {, or } 81455) \text {. }\end{array}$ \\
\hline
\end{tabular}

If the analyte tested is not listed under one of the Tier 2 codes or is not represented by a Tier 1 code in CPT, use of the unlisted CPT code 81479 is required.

Advises gene sequencing CPT codes do not categorize tests with the detail needed to determine medical necessity and requires a "Z-Code" identifier specific to the applicable test as additional claim documentation. Palmetto GBA reviews a comprehensive

Palmetto GBA (NC, SC, VA, test/assay dossier inclusive of clinical information to determine if a test meets Medicare's reasonable and necessary requirement Prior to a "Z-Code" identifier, Palmetto will consider all claims for these tests on an individual consideration basis.

Noridian (AZ, ID, AK, MT, ND, OR, SD, UT, WA, WY, CA, HI, $\mathrm{NV}$ ), CGS Administrators (KY, $\mathrm{OH})$, and First Cost (FL, USVI)

Have indicated they will also require a Z-Code for Molecular Diagnostic claims submissions. Individual labs performing genomic tests are required to apply for Z-Code identifiers for inclusion on claims for molecular diagnostic and genetic tests. 
Table 3. Private payor policy/coverage statements for expanded panel testing

\begin{tabular}{|c|c|}
\hline Priva & e Statement \\
\hline $\begin{array}{l}\text { Anthem Blue Cross } \\
\text { Blue Shield \& BCBS } \\
\text { of Georgia }\end{array}$ & $\begin{array}{l}\text { Effective January 1, } 2015 \text { policy titled, "Molecular Profiling for the Evaluation of Malignant Tumors” reads, "Profiles include evaluation of } \\
\text { multiple genes through gene expression analysis, gene sequencing and other techniques. These profiles catalogue a number of genetic markers in } \\
\text { an attempt to select optimal therapy.” It further states, "Molecular profiling as a method to guide the selection of therapeutic agents for malignant } \\
\text { tumors is considered investigational and not medically necessary.” Under this policy, CPT Codes 81445, 81450, 81455, and 81599 (unlisted } \\
\text { multi-analyte assay with algorithmic analysis), (when specified as molecular profiling for malignant tumors, e.g. Foundation One, Molecular } \\
\text { Intelligence Service (Target Now), GeneKey or OncInsights) are not covered because they are investigational and not medically necessary. }{ }^{[13]}\end{array}$ \\
\hline BCBS of Illinois & $\begin{array}{l}\text { Effective January 1, } 2015 \text { policy titled, "Molecular Panel Testing of Cancers to Identify Targeted Therapies” reads, "The use of expanded cancer } \\
\text { mutation panels for selecting targeted cancer treatment is considered experimental, investigational and/or unproven.” This policy is intended to } \\
\text { address expanded panels that test for many potential mutations that do not have established efficacy for a specific cancer in question. The policy } \\
\text { does not apply to individual markers that have demonstrated efficacy and specifically mentions HER2 (ERBB2) for Breast Cancer, KRAS and } \\
\text { BRAF c1799T > A for Colon Cancer, EGFR and ALK/ROS1 for NSCLC, BRAF V600 for Metastatic Melanoma, BRC-ABL for CML, and KIT } \\
\text { for stromal tumors. Additionally, this policy gives “consideration” for multi-analyte panels limited to a particular type of cancer such as NSCLC. } \\
\text { FoundationOne }{ }^{\mathrm{TM}} \text {, Foundation OneHeme, Illumina Inc. (TruSeq }{ }^{\circledR} \text { Amplicon and TruSight }{ }^{\mathrm{TM}} \text { Tumor Panels), Life Technologies (Ion } \\
\text { AmpliSeq }{ }^{\mathrm{TM}} \text { cancer panels and the Ion AmpliSeq Cancer Hotspot Panel) are considered genetic panels for a large number of cancer-associated } \\
\text { mutations for which the evidence is insufficient to make any conclusions on clinical utility. "In addition, there is potential for harm if ineffective } \\
\text { therapy is given based on test results, because there may be adverse effects of therapy in absence of a benefit. As a result, the use of expanded } \\
\text { mutation panel testing (81445, 81450, and 81455) for targeted treatment in cancer is considered experimental, investigational and/or } \\
\text { unproven." }\end{array}$ \\
\hline Aetna & $\begin{array}{l}\text { Policy } 0532 \text { “Tumor Markers” last reviewed October 23, } 2015 \text { identifies } 51 \text { tumor markers covered for specified indications and } 93 \text { tests } \\
\text { considered experimental and investigational with insufficient peer-reviewed literature to support these tests in a clinical setting. The list of } \\
\text { experimental tests includes the Caris Target Now Molecular Profiling test (\#14), Colonext Next-Gen Cancer Panel (\#21), the FoundationOne and } \\
\text { FoundationOne Heme (\#37), Ovanext Next-Gen Cancer Panel (\#58), and total (whole) gene sequencing for cancer (\#87). }{ }^{[15]}\end{array}$ \\
\hline CIGNA & $\begin{array}{l}\text { Effective January 15, } 2015 \text { policy titled, "Tumor Markers for Cancer" includes a table of biomarkers that are covered for specified conditions. The } \\
\text { policy expressly denies coverage for multi-gene expression testing (including FoundationOne) and tumor profiling (including Caris Target } \\
\text { Now }{ }^{\mathrm{TM}} \text { ) because these tests are experimental, investigational, or unproven. Interestingly, the unlisted molecular pathology procedure code } 81479 \\
\text { is identified as not covered, however the targeted molecular sequencing codes (81445, 81450, and 81455) are not addressed in this policy. }{ }^{[16]}\end{array}$ \\
\hline
\end{tabular}

(1) Billing the government for targeted genomic panels using CPT 81455 may be a false claims risk given the non-coverage decision published by many of the MACs. Using CPT Code 81455 (the code that most accurately describes the service ordered and provided) may be reasonable if the Medicare claim is annotated to identify the panel as a noncovered service submitted at the beneficiary's insistence to obtain a coverage determination.

(2) Performing and reporting comprehensive gene/genomic panels that meet the 81455 coding description and using the tiered coding methodology to bill may be a false claims risk in that billing is not consistent with the services provided. (The NCCI identifies "unbundling" [using multiple procedure codes to bill for a group of procedures that are covered by a comprehensive code] as inappropriate when used as an intentional strategy to maximize payment with potential false claims implications.)

(3) Considerable administrative burden associated with billing using the tiered coding methodology in com- pliance with the LCDs issued by MACs in each of the respective billing jurisdictions.

(4) The quantified business impact of a government payor population within the overall payor mix.

(5) The potential to bill Medicare patients directly for non-covered NGS services.

(6) Implications of "balance billing" or billing patients for the balance owed after Medicare has reimbursed the claim. While it is an expectation that patients will be responsible for copays and deductibles associated with covered services, balance billing is illegal when the provider accepts Medicare assignment, and/or the amount exceeds a Medicare negotiated rate. Balance billing is also illegal when billing items and services deemed non-covered to Medicare as a secondary payor when a private payor is primarily responsible for paying but denies reimbursement.

Multiple MACs have indicated CPT code 81455 is excluded from coverage and will be denied. A decision to bill Medicare using individual MoPath Tier 1 and Tier 2 codes for tests deemed medically necessary by the MAC in the respective 
billing jurisdiction must be in strict adherence to the relative LCD, and when available use the Z-code identifier for those MAC jurisdictions requiring this information on the claim. Billing tests with approved Z-Codes, FDA approved companion diagnostic tests, MAAAs with MAC coverage approval/codes, and NSCLC testing using 81455 may be considered lower risk so long as there is evidence of medical necessity in patient records. An advanced beneficiary notice of non-coverage $(\mathrm{ABN})$ may be used to notify patients in writing that Medicare may not pay for molecular tests.

\subsection{Private payors}

Billing private payors for targeted genomic/NGS panels is primarily a business discussion to determine the most efficient coding strategy with the best FMV reimbursement for the services. Some private payors have communicated non-coverage policy statements for molecular profiling as a method to guide the selection of therapeutic agents for malignant tumors because the testing is considered investigational and not medically necessary. Options for billing may include:

(1) Using CPT Code 81455 anticipating a denial and leveraging the appeals process to collect reimbursement for testing supported as medically necessary justified by authoritative sources (clinical utility) and evidence of analytical (CLIA certified/CAP accredited) and clinical validity (rare or aggressive disease, disease that has previously failed other treatments).

(2) Using the tiered coding methodology to bill for the comprehensive panel using an appropriate unit of service for molecular pathology procedure level codes 81403-81408 (Tier 2 codes) and using the unlisted code 81479 if the biomarker is not specified and/or the services performed do not fall under a Tier 1 or Tier 2 code.

Using tiered codes to identify all of the gene/genomic testing performed using NGS technology, even those genes "for which the potential value of outcomes has not yet been proven", aligns more closely with billing for the services provided.

Supporting logic for this methodology includes:

- Large panel testing technology has evolved faster than the clinical outcomes data evaluating efficacy of such testing, and the published coverage decisions lag behind the evolution of clinical outcome data.

- Leveraging the full capacity of NGS technology to perform multiple tests with one sample is a responsible and efficient use of valuable,scarce or small tissue samples.
- Reporting all drug/biomarker results equips the physician with information known to influence positive patient outcomes as well as information that, while it may not be "proven", may be valuable for treatment decision in the practice of medicine.

- This coding strategy used to bill for the services performed/provided optimizes reimbursement.

(3) Using the tiered coding methodology to bill only medically necessary biomarkers as cited by authoritative evidence such as NCCN or ASCO guidelines, Novitas LCDs (L34796/L33638) or Cigna coverage Policy 0172. Those tiered codes would correlate to allowable ICD 10 diagnosis codes and be billed using units of service (UOS) correlating to the number of medically necessary genes analyzed within the Tier 2 codes. This methodology may impose additional administrative burden and result in lower reimbursement, however, it aligns more closely with billing for covered services.

When considering options 2 or 3 , the business discussion should determine not only how to properly use the UOS to bill for Tier 2 codes but also to determine appropriate pricing for each Tier 2 code:

- One way is to bill a unit of service for each gene that is tested using the appropriate Tier 2 code that describes the level of technical resources and professional interpretive work and use UOS as a multiplier times a fixed price for each Tier 2 code (not to exceed the maximum allowable so as to avoid triggering medically unnecessary edits or MUEs) - example billing 81405 eleven (11) times at $\$ 500$ for an analysis of 11 genes each with 11-25 exons (using the code to bill for each gene tested).

- Another way is to bill "what the market will bear" (81406 bears \$10,396 from BCBSAZ) using the appropriate code that describes the level of technical resources and professional interpretive work and billing only one UOS regardless of the number of genes analyzed (using the code to bill a panel within a panel).

(4) Using another or a hybrid methodology as recommended by consultants/experts with domain knowledge in billing, coding and reimbursement.

\section{Discussion}

Trosman et al. conducted interviews with NGS experts and 10 major payors representing 125,000,000 enrolled private payor beneficiaries to better understand the challenges of 
reimbursement decisions. (Trossman, 2015) They concluded, "Payors perceive that NGS holds significant promise but, in its current form, poses disruptive challenges to coverage policy frameworks." ${ }^{[18]}$ In their study, $80 \%$ of payors struggle to qualify NGS as medically necessary, and $70 \%$ are skeptical of evidence methods used in NGS reporting. Payors base reimbursement decisions on reliable, reproducible, clinically valid tests and are concerned about the variability between laboratories, test platforms and variant interpretation, resulting in conservative coverage and reimbursement policies for molecular testing including NGS. Private payors consider FDA approval as an indicator for coverage eligibility and as such may have policy considerations for FDA approved testing (e.g. companion diagnostics).

Arguably, there are different platforms and assays for NGS, different annotation and interpretation schemes, and different instruments have different sensitivity and specificity. Even when tests have the same sensitivity and specificity metrics, the tests may be fundamentally different in terms of the breadth of the mutations (amplification vs. hybrid capture; or differing bioinformatics used to annotate the variants). Yet, NGS is a powerful technology with a capacity to process DNA sequencing much faster, exhausting less tissue and at a lower cost than traditional methods. The testing may identify DNA sequence variations, both acquired (somatic) as well as inherited (germline) mutations that could define therapeutic targets for treatment that may improve patient outcomes. The use of multi-plex or multi-gene panel testing such as NGS is expanding faster than the scientific evidence; however, some genomic panels (81445) have been identified as covered, specifically for NSCLC; and tiered coding options allow payment for medically necessary tests performed using NGS technologies. For example, Anthem Blue Cross Blue Shield covers BRAF V600E mutation analysis (for individuals with unresectable or metastatic melanoma to select those who would benefit from treatment with an FDA-approved BRAF inhibitor) using tiered MoPath coding, even if the test was performed using NGS, rather than the Cobas 4800 System. ${ }^{[13]}$

While panel testing is time and cost efficient, minimally disruptive for patients and physicians, and spares valuable specimens, payors struggle with reimbursing for genes not necessarily linked to the disease for which the test is being billed based on current definitions of medical necessity for the tests and clinical utility of the results. As payors consider medical necessity of the expanded panels for coverage and reimbursement of the new GSP CPT codes, the potential for classification as experimental/investigational is likely and will most typically result in a negative coverage statement.
As the private payors, as well as Medicare, continue the trend of applying evidence-based approaches to the determination of coverage, payors will seek to establish coverage policy only for those tests that demonstrate results leading to improved clinical decisions and where actions result in improved patient outcomes. The Center for Medical Technology Policy (CMTP) draft guidelines for NGS Oncology Testing suggest that payors should rely on the CAP accreditation program and proficiency testing to ensure analytic validity of NGS panels. ${ }^{[19]}$ Leveraging CLIA certification and CAP accreditation may support analytic validity of a test in an appeal for payment. Clinical utility refers to the usefulness of the test and the value of information to medical practice and represents a balance between health-related benefits and potential harms that may occur from a test result or the use of the result to determine patient treatment. While the data to support clinical utility of NGS panel testing may be limited, there may be an argument that the benefits (mortality/survival; quality of life) of the testing results in treatment decisions may outweigh the potential harms at least for certain populations where "current management" is either exhausted or not defined. In their draft policy guidelines, the CMTP suggests payors should cover panels larger than 50 genes (CPT code 81455 ) for certain patients including "those with newly diagnosed stage IV lung adenocarcinoma, newly diagnosed carcinoma of unknown primary origin, newly diagnosed stage IV rare or uncommon tumor for which no standard treatment exists, newly diagnosed stage IV tumors with a median overall survival of less than two years, stage IV solid tumors who have exhausted established guidelines for treatment, or newly diagnosed hematologic malignancies with limited established treatment guidelines" (GenomeWeb, 2015). Establishing clinical validity for molecular tests is limited by the rapid evolution of technology and science such that all main causative mutations may not be known, and there is no "gold standard" molecular test for conditions caused by more than one gene or by more than one variant within genes. Clinical validity is evidenced by responders to targeted therapies, however gathering the statistically significant data is an ongoing challenge. CMTP guidelines support coverage of NGS panels consisting of five to 50 genes (CPT codes 81445 or 81450 ) when such panels include at least five genes supported as routine care and medically necessary by authoritative evidence.

Reimbursement for the full capacity of NGS technology to perform multiple tests with one sample as the responsible and efficient use of valuable tissue is highly variable; and while not all of the reported NGS results and drug/biomarker associations may be "proven", pharmacogenomics equips physicians with information valuable in the practice of per- 
sonalized medicine. Submission of a claim, in any case, is important in order to obtain appeal rights and to preserve rights to re-bill according to payor policies. Understanding the applicable laws and policies allows leaders to make decisions about their NGS billing strategy with considerations for revenue impact, regulatory exposure and risk tolerance.

\section{Conclusions}

At the time of this writing, the 2016 CMS CLFS clinical lab fee schedule and new CPT codes released on December 15, 2015 , indicate that in spite of the complexity of genomic testing, reimbursement may be catching up with published evidence associated with genomic testing. New CPT codes have been issued for limited gene panel testing for hereditary cancer and cancer-related disorders, and GSP codes 81450 (\$648.40) and 81445 (\$597.91) have been assigned payment limits/midpoints on the 2016 CLFS. ${ }^{[20]}$ While this is encouraging, we are mindful that codes and a payment amount are not indicative of established medical necessity, affirmative coverage or guaranteed payment. There is coverage for tests proven to lead to immediate actionable therapy, tests proven to inform treatment, and tests proven to have utility in treating genetic disease. Coverage for large NGS panels however, is still complicated by the reporting of analytes that are proven (covered) combined with analytes for which there is insufficient evidence (noncovered). A "less is more" reimbursement model that requires evidence of clinical utility does not currently value a "more is better" genomic dataset that may inform the practice of personalized medicine. And as such, it remains a challenge to submit the right claim for the right gene and the right indication in order to avoid billing risk and obtain payment. Having sufficient information and background around the reimbursement landscape in the field of genomics is essential for strategic billing and claims submissions in the current and evolving billing climate.

\section{REFERENCES}

[1] Zweifel R. Review and project methods for CPT code assessment GEM cancer panel. St. Paul, MN: Panacea Healthcare Solutions, Inc; 2014.

[2] BCBS. MED208.061 Molecular Panel Testing of Cancers to Identify Targeted Therapies. Blue Cross Blue Shield of Illinois Coverage Policy. 2015.

[3] Novitas Solutions I. LCD Biomarkers for Oncology (L35396). Centers for Medicare and Medicaid Services Coverage Database. 2015.

[4] CMS. Centers for Medicare and Medicaid Services Coverage Database. 2015.

[5] Social Security Act. 1862 (a)(1)(A). (n.d.).

[6] FDA. FDA Overview of IVD Regulation. 2015.

[7] Department of Health \& Human Services. FDA Document Number GEN1500674. Silver Spring, MD. 2015.

[8] FDA. Guidance for Industry, Food and Drug Administration Staff, and Clinical Laboratories Framework for Regulatory Oversight of Laboratory Developed Tests (LDTs). FDA Draft Guidance. 2014.

[9] Roche Molecular Systems, Inc. FDA Device Approvals, Denials, and Clearances. FDA. 2011. Available from: http://www. accessda ta.fda.gov/cdrh_docs/pdf11/P110020c.pdf

[10] Genentech. FDA Approved Drug Products. FDA. 2011. Available from: http://www.accessdata.fda.gov/drugsatfda_docs /label/2011/202429s0001bl.pdf

[11] CMS. Medicare National Coverage Determinations (NCD) Coding Policy Manual and Change Report. Centers for Medicare \& Medicaid Coverage Center. 2013. Available from: https://www.cms.gov/Medicare/Coverage/CoverageGenI nfo/Downloads/manual201301.pdf

[12] OCR. Office of Civil Rights Health Insurance Portability and Accountability Act of 1996 . Public Law. 1996.

[13] Anthem BCBS. Coverage Policy GENE 00025 Molecular Profiling for the Evaluation of Malignant Tumors. Anthem Blue Cross Blue
Shield. 2015. Available from: https://www . anthem. com/ca/me dicalpolicies/policies/mp_pw_c150728.htm

[14] BCBS Il. Blue Cross Blue Shield of Illinois Coverage Policy MED208.061 Molecluar Panel Testing of Cancers to Identify Targeted Therapies. Blue Cross Blue Shield of Illinois. 2015. Available from: http://www.medicalpolicy.hcsc.net/med icalpolicy/activePolicyPage?lid=i3ed71ty\&corpBrand =Boeing\&corpEntCd=IL1

[15] Aetna. Policy 0352 Tumor Markers. Aetna. Available from: http: //www.aetna.com/cpb/medical/data/300_399/0352.html

[16] Cigna. Cigna Coverage Policy 0172 Tumor Markers for Cancer. Cigna. 2015. Available from: https: //cignaforhcp. cigna.com/public/content/pdf/cov eragePolicies/medical/mm_0172_coveragepositioncrit eria_tumor_markers_for_diagnosis_mgmt_cancer.pdf

[17] Caris MPI, Inc. Supplementary Medical Insurance Benefits, M-12648 (Department of Health and Human Services Departmental Appeals Board April 24, 2012).

[18] Trossman JW. Challenges of coverage policy development for nextgeneration tumor sequencing panels: experts and payors weigh in. Journal of the National Comprehensive Cancer Network March. 2015; 13(3): 311-318.

[19] CMTP. Initial Medical Policy and Model Coverage Guidelines for Clinical Next Generation Sequencing in Oncology Report and Recommendations. Retrieved from Green Park Collaborative of the Center for Medical Technology. 2015. Available from: http://www.cmtpnet.org/docs/resources/Full_Re lease_Version_August_13

[20] CMS. 2016 Clinical Diagnostic Laboratory Fee Schedule. Retrieved from Centers for Medicare \& Medicaid. 2015. Available from: https://www.cms.gov/Medicare/Medicare-Fee-for -Service-Payment/ClinicalLabFeeSched/clinlab.html 